\title{
Associating Assertions with Business Processes and Monitoring their Execution
}

\author{
Alexander Lazovik \\ ITC-IRST \\ and \\ DIT, University of Trento \\ 38050 Trento, Italy \\ lazovik@dit.unitn.it
}

\author{
Marco Aiello \\ DIT \\ University of Trento \\ Via Sommarive, 14, 38050 \\ Trento, Italy \\ aiellom@dit.unitn.it
}

\author{
Mike Papazoglou \\ Infolab \\ Tilburg University \\ PO Box 90153, NL-5000 LE, \\ The Netherlands \\ mikep@uvt.nl
}

\begin{abstract}
Business processes that span organizational borders describe the interaction between multiple parties working towards a common objective. They also express business rules that govern the behavior of the process and account for expressing changes reflecting new business objectives and new market situations.

In our previous work we developed a service request language and support framework that allow users to formulate their requests against standard business processes. In this paper we extend this approach by presenting a framework capable of automatically associating business rules with relevant processes involved in a user request. This framework plans and monitors the execution of the request against services underlying these processes. Definitions and classifications of business rules (named assertions in the paper) are given together with an assertion language for expressing them. The framework is able to handle the non-determinism typical for service-oriented computing environments and it is based on the interleaving of planning and execution.
\end{abstract}

\section{Categories and Subject Descriptors}

H.3.5 [Information Storage And Retrieval]: Online Information Services-Web-based services; I.2.8 [Artificial Intelligence]: Problem Solving, Control Methods, and SearchPlan execution, formation, and generation

\section{General Terms}

Verification, Algorithms, Design

\section{Keywords}

Service delivery, monitoring, quality, and management, Theoretical frameworks for service representation and composition, Service and AI Computing

Permission to make digital or hard copies of all or part of this work for personal or classroom use is granted without fee provided that copies are not made or distributed for profit or commercial advantage and that copies bear this notice and the full citation on the first page. To copy otherwise, to republish, to post on servers or to redistribute to lists, requires prior specific permission and/or a fee.

ICSOC'04, November 15-19, 2004, New York, USA.

Copyright 2004 ACM 1-58113-871-7/04/0011 ...\$5.00.

\section{INTRODUCTION}

Web services technologies offer high-level specifications that provide functionality that supports and leverages web services and enables specifications for integrating automated business processes. Currently, there are two largely complementary initiatives for developing business process definition specifications which aim to define and manage business process activities and business interaction protocols comprising collaborating web services. The terms orchestration and choreography have been widely used to describe business interaction protocols comprising collaborating web services. Orchestration (as championed by BPEL) describes how web services can interact with each other at the message level, including the business logic and execution order of the interactions from the perspective and under control of a single endpoint. Orchestration refers to an executable business process that may result in a long-lived, transactional, multistep process model. Choreography (as championed by the Web Services Choreography Description Language) is typically associated with the public (globally visible) message exchanges, rules of interaction and agreements that occur between multiple business process endpoints, rather than a specific business process that is executed by a single party. Choreography is more collaborative in nature than orchestration. It is described from the perspectives of all parties (common view), and defines the complementary observable behavior between participants in business process collaboration. Currently, both orchestration and choreography initiatives are in their infancy and based on WSDL which is strongly emphasizes XML syntax and human-targeted descriptions.

In this paper we propose an approach based on interleaving planning and execution in the context of non-deterministic domains to deal with user expressed requests against standard business processes that result in initiating and executing business processes from diverse organizations. The execution of these business processes in the proposed framework is governed by assertions, which are business rules applied to processes. The framework we propose deals with non-deterministic domains, where it tries to satisfy a user request by taking into account how assertions that appear at different levels, e.g., business process, role, and provider level, are applied during business process execution. The framework focuses in particular on the application of business rules that are associated with choreographies. The ap- 
plication of choreography assertions usually results in activating only selected business process segments in different organizations. These are the business process segments that satisfy the choreography constraints and consequently can be involved in the result of a user request. In addition, the execution path of business processes is monitored to make certain that environmental conditions, i.e., web service supplied information, conform to the choreography assertions and user request requirements. The proposed framework deals with three kinds of assertions depending on their operational context and complexity: simple assertions, where simple reachability conditions are checked; preservation assertions, where maintenance of some condition needs to be satisfied throughout a path comprising a set of states traversed by the process during execution time; and business entity assertions, where the evolution sequence of a particular variable is monitored for correctness. In this paper we are not concerned with the effect that choreography assertions have on orchestration assertions (assertions that apply in the local context of an organization). We henceforth use the term assertion to mean choreography assertions.

The remainder of the paper is organized as follows. In Section 2 , we recall the notion of the service-oriented business process and introduce various kinds of assertions. Section 3 presents an interleaving planning and execution framework for the monitoring of the execution of user requests and assertions against standardized business processes. Section 3.1 presents a formal domain definition. Section 3.2 introduces the service assertion language XSAL. A formulation of an example in terms of the formally defined domain is the offered in Section 3.3; while Section 3.4 provides algorithms for the working of the framework. Section 4 illustrates how the proposed framework processes the assertions on a traveling marketplace example. Related work is overviewed in Section 5. Concluding remarks and future work are discussed in Section 6.

\section{BUSINESS PROCESSES AND ASSERTIONS}

A process is an ordering of activities with a beginning and an end; it has inputs (in terms of resources, materials and information) and a specified output. We may thus define a process as any sequence of steps that is initiated by an event, transforms information, materials, or business commitments, and produces an output [6]. In this paper, we consider business processes as a means to represent the control flow of business logic and applications. This is achieved by introducing the notion of a state and an action. A state represents the state of the process execution. An action represents a business activity, which is modeled as a transition between given states. Each action is executed on behalf of a role. A role represents a set of business operations that relate to the same party, e.g., a travel agency. Each role has a number of providers associated with it. The providers can be found by interacting with service registries, e.g., UDDI. A provider is the actual party that implements a role, e.g., a specific travel agency. It is convenient to also define the notion of a process variable, which is a variable associated with a process changing values, e.g., travel packages, hotel reservations, etc, as the process progresses through its execution path and its states change. The use of process variables guarantees that the execution of a business process can be monitored during execution as the process traverses a set of states where constraints may need to be applied to these variables. Constraints on the variables may represent user request or business rules.

\subsection{An example in the traveling marketplace}

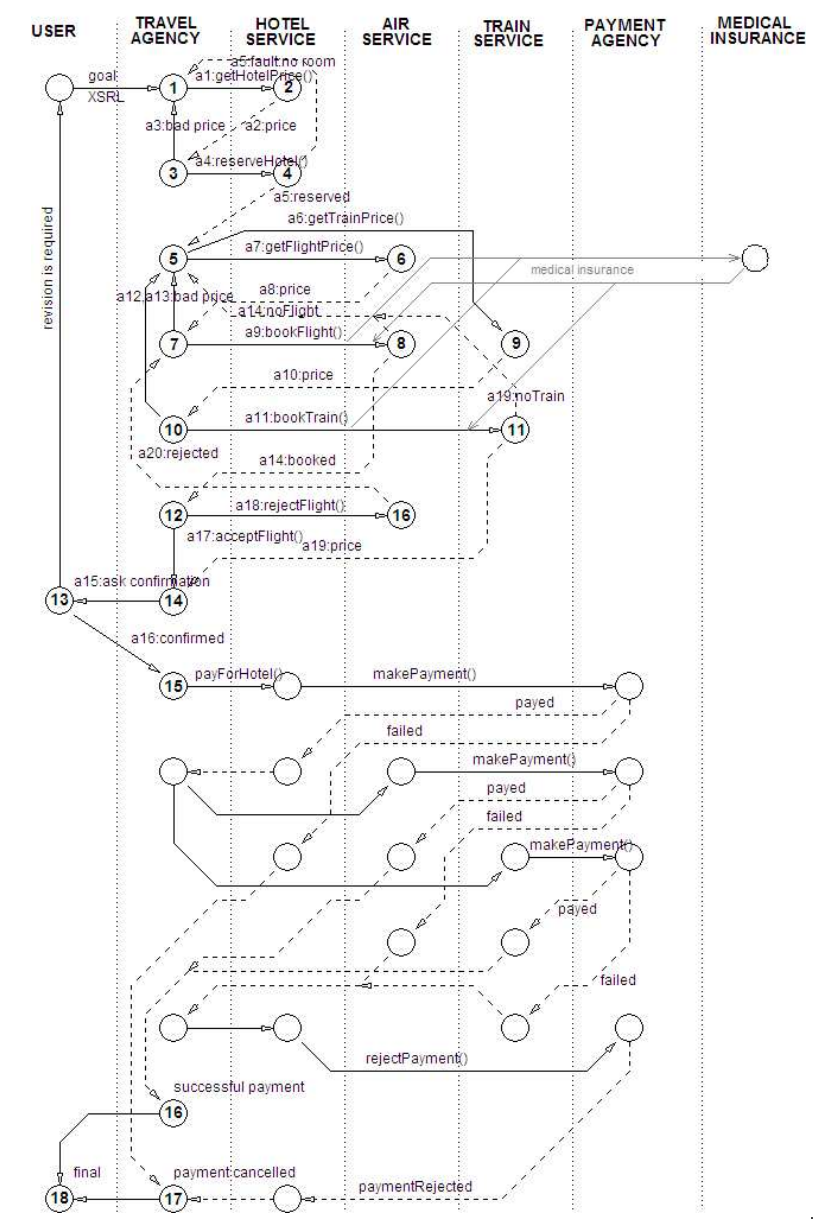

Figure 1: A traveling business process.

Consider a user requesting a trip to Nowhereland and having a number of additional requirements regarding such a trip, e.g., that the total price of the trip be lower than 300 euro, the prices of the hotel below 200 euro, avoid using the train, and so on. To be satisfied such a request involves the interaction with various autonomous service providers, including a travel agency, a hotel booking company and a flight carrier. The services reside in the same travel marketplace and must follow a standard business process for that domain such as the one exemplified in Figure 1. This process is modeled as a state transition diagram, that is, every node represents a state in which the process can be, while labeled arcs indicate how the process changes state. Actors involved in the process are shown at the top of the diagram. The actors include the user, a travel agency, a hotel service, an air service, a train service and a payment service.

The process is initiated by a user contacting a travel agency, hence, (1) is the initial state. The state is then changed to $(2)$ by requesting a quote from an hotel (action $a_{1}$ ). The dashed arcs represent web service responses, in particular arc $a_{2}$ brings the system in the state (3). The execution 
continues along these lines by traversing the paths in the state transition diagram until we reach state (14). In this state a confirmation of a hotel and of a flight or train is provided by the travel agency and the user is prompted for acceptance of the travel package (13).

The state transition diagram is non-deterministic. This is illustrated, for instance, in state (4). In this state the user has accepted the hotel room price, however, is faced with two possible outcomes, one that a room is not available (where the system transits back to state (1)) and the other where a room reservation can be made (state (5)). The actual path will be determined at run-time when appropriate services provide information regarding how availability for the hotel providers is chosen.

The lower part of the business process models the payment of the travel package just booked as an atomic action. This means the entire trip is payment atomic.

Services involved in the above process may have additional requirements and business rules that need to be followed. A particular travel carrier may require advanced payment, a travel agency may want to always have explicit user's approval before committing to a package. At a higher level, different marketplaces may implement the same process but with different rules. For instance, one may additionally require that all air carriers use a specific communication protocol. This sort of additional business rules are called assertions and are defined next.

\subsection{Assertions}

Actions of a business process are usually extend across different parties (organizations which may play different roles) that are involved in different fragments of the process. A choreography language can guarantee the consistency of service interfaces, message ordering and message invocations but it can not be used to check process runtime properties. Safe execution of the business process can only be ensured by a monitoring mechanism that checks the run-time properties of business process and possibly recovers it from assertion violations. The monitoring of the business process based on observing their assertions is performed in the following way. First, assertions are published by the party which wants its assertions to be applied to business processes and monitored during execution. When executing the business process, the framework allows only those executions to proceed where published assertions are satisfied. If an assertion is violated then the system tries to find an alternative execution path in the business process that does not violate the assertion, if any. Assertions are published on different levels: business process, role or provider. During execution, assertions defined on the business process level are always taken into account; assertions defined by roles are checked only if operations for that role are invoked; provider level assertions are considered if an action of the particular provider is necessary.

More precisely, monitoring is a mechanism that ensures the execution of a process is consistent with respect to choreography business rules and user specified requests. As a business process spans several organizations, all of them expect that their business rules are taken into account when executing the process. Business rules are supplied by service providers and are enforced on business processes that are associated with such rules during their execution.

Business rules are expressed in the context of a process

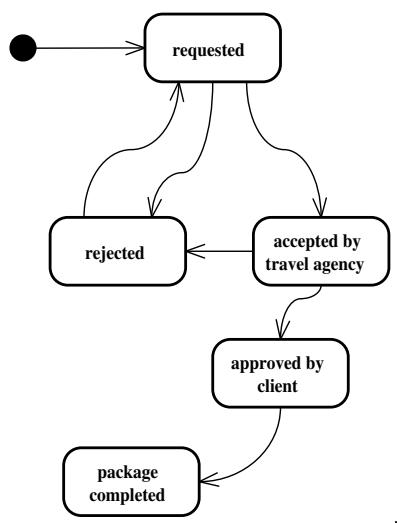

Figure 2: A travel package business entity assertion.

by assertions. An assertion may be either satisfied or not during the execution of a business process. An assertion may be defined formally as follows:

Definition 1. An assertion is a condition that applies over the execution of a business process. Given a business process and a specific state, we say that an assertion is satisfied if the assertion is true in its current state and in all future states visited during process execution. In the following we use the term assertion and business rule interchangeably.

We may classify assertions along two different dimensions: (i) operational assertions: on the basis of the operational context and complexity of the assertion; (ii) actor assertions: on the basis of the ownership of the assertion.

There are three types of operational assertions that can be distinguished on the basis of the above classification:

Simple assertion. A simple assertion is a condition to be satisfied in a given state or a specific set of states in order to reach a successor state. Simple assertions are also named reachability assertions. An example of such an assertion in the context of a traveling domain is the requirement for having a medical insurance if the period of being abroad is more than two weeks. To comply with this assertion we must ensure that if the client requests a travel package with duration beyond two weeks then a medical insurance must be obtained before the business process progresses successfully.

Preservation assertion. A preservation assertion is a condition to be maintained throughout all states reached during the execution of a business process. Preservation assertions are also named maintainability assertions. In the same traveling example, consider a situation where special offers exist for clients who hold a frequent flyer royalty card, e.g., OneWorld. An assertion for the use of such card would require that all invoked services accept the card to provide discount or points. To comply with this assertion the execution of the business process will attempt to maintain the execution on those paths where services adhering to the royalty program are available.

Business entity assertion. A business entity assertion is a property that applies to the evolution sequence of 
a process variable during process execution. For instance, a business entity assertion can be associated with the status of a travel package, see Figure 2. Initially, the "status" variable must assume the value "requested' when the travel package operation is started. From this state, the request can be 'rejected' if the traveling agency fails to satisfy it and, eventually, return in a 'requested' status. Alternatively, the status variable can be 'accepted by travel agency' and subsequently be 'approved by client' and finally become a 'package completed'. To comply with this assertion the execution of the business process must ensure that the states of the travel package variable are reached in the prescribed sequence and only change value according to the valid states of the business entity assertion described above.

Assertions are not only classified on the basis of their operational dimension but also on the basis of ownership. Based on this criterion we may distinguish between different levels of assertion ownership , see Table 1.

Business process-level. The business process level assertions are applied to the whole business process. The business process execution environment verifies these assertions during all executions and for all used services. Assertions of this type are maintained by the party who defines the choreography message sequences. These assertions are stored together with the business process itself. The business entity assertion defined in Figure 2 is an example of business process level assertion. It defines possible state transitions for the travel package for all execution sequences in the business process. Another example is the following. Usually business processes have an assertion of always reaching the final state despite of the nondeterminism inherent in dealing with web service implementations, e.g., purchase a travel package. This assertion ensures process consistency with organization rules and policies.

Role-level. Role-level assertions that are valid for all the providers undertaking a specific role. Typically these assertions represent the constraints defined by standard organizations, government, etc. For example, due to governmental laws all travel agencies may require that together with a flight ticket also a medical insurance is purchased, whenever the final destination is in a particular set of geographic locations where health risks may exist. These assertions are defined together with the service interfaces and stored together with the service descriptions.

Provider-level. At the lowest abstraction level assertions are published by a particular service provider. These assertions are stored in service registries together with service implementations. Provider-level assertions are used when a particular provider wants to enforce internal consistency of the business process by making the process observe its own business rules at runtime. For instance, provider role assertions may involve payment service providers having additional constraints, such as, protocol communication preferences, organization licensing, authentication, etc.

\section{MONITORING FRAMEWORK}

One of the biggest challenges that web service enabled emarketplaces face is the lack of support for appropriate service request languages that retrieve and aggregate services that contribute to the solution of a business problem. Users typically require services from an service-based marketplace on the basis of service characteristics and functionality as supplied by service providers. A service request language provides for a formal means of describing desired service attributes and functionality, including temporal and nontemporal constraints between services, and service scheduling preferences.

Our previous research work concentrated on developing a service request language for web services in service-oriented marketplaces that contains a set of appropriate constructs for expressing requests and constraints over requests as well as scheduling operators $[1,13,9]$. This language is named XSRL for XML Service Request Language. XSRL was introduced in $[1,13]$ and enables a user to formulate complex requests against standard business processes. These standard processes are provided by a market maker (a consortium of organizations) that brings customers, suppliers and vendors together. The market maker assumes the responsibility of creating a service-marketplace administration and performs maintenance tasks to ensure the administration is open for business and, in general, provides facilities for the design and delivery of business processes that meet specific business needs and conforms to industry standards [14]. Standard business processes are described in a choreography language such as Web Services Choreography Description Language (WS-CDL) [7]. WS-CDL specifies the common observable behavior of all participants engaged in business collaboration. Each participant could be implemented by completely different languages such as web services applications, whose implementation is based on executable business process languages like BPEL, XPDL and BPML.

XSRL expresses a request and executes it according to the user preferences. The framework that takes XSRL request as input returns a product as the result of the request, e.g., constructs an end-to-end holiday packages (documents) comprising a number of flight and accommodation choices. XSRL is equipped with constructs for expressing quantitative requests, such as, booking of a room for two nights, spending between 100 and 200 euro, etc., but also qualitative operations for sequencing goals, such as, booking a room only after having booked a plane, for stating preferences, e.g., flying rather than taking a train to a destination, for stating the maintaining of a condition during execution, such as, keeping the budget below 500 euro. Loosely speaking, the response documents can be perceived as a series of plans that potentially satisfy a request. In expressing an XSRL request it is important that a user is enabled to specify the way that the request needs to be planned and executed. We refer to [9] for the syntax, semantics and detailed examples of XSRL.

XSRL and its supporting framework are a powerful tool for enabling a user to formulate requests against business processes but it currently lacks support for choreography assertions supplied by service providers and/or market makers that can be associated with the execution of a choreographed process. Assertions are essential means for the actors delivering the services and market makers to apply enterprise/marketplace policies and conditions. In this pa- 


\begin{tabular}{|c||c|l|}
\hline Assertion level & Location stored & Usage \\
\hline \hline business process & domain description & concatenated with user request \\
\hline role & service description & applied if an action of the role is invoked \\
\hline provider & service registry & applied if the provider is associated with an action to be invoked \\
\hline
\end{tabular}

tion levels.

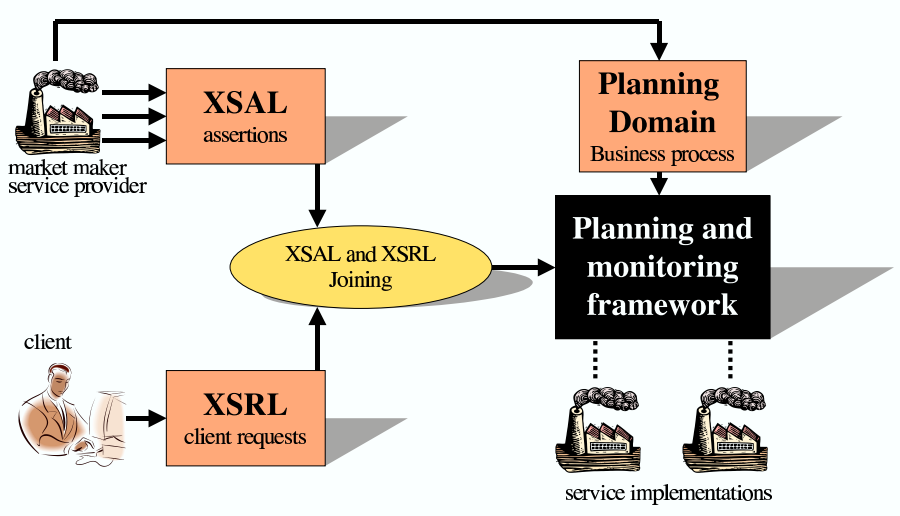

Figure 3: Handling of XSAL and XSRL requests.

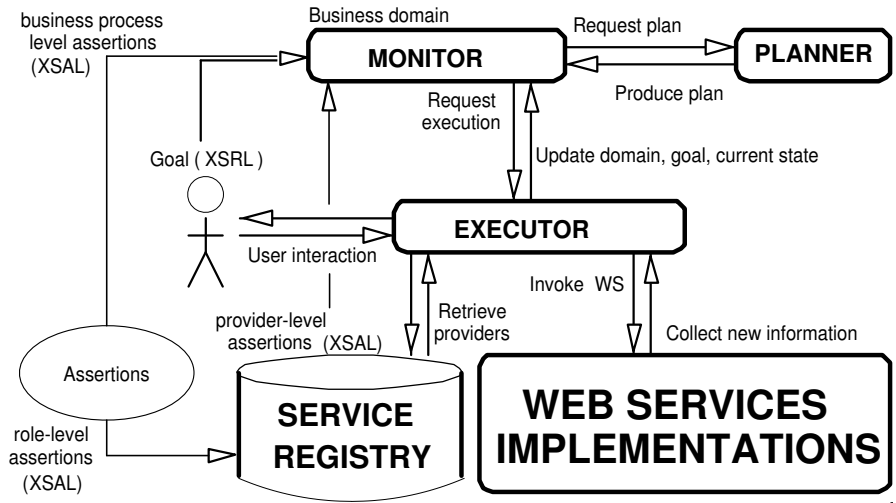

Figure 4: Planning and monitoring framework. per we address this limitation of XSRL by demonstrating how the language and its support environment is extended by means of an assertion language, which we name XSAL (XML Service Assertion Language). This language is defined in Section 3.2.

XSRL and XSAL work in tandem during the planning and monitoring of business processes in order to satisfy the user requests in conjunction with applying service provider and marketplace maker supplied assertions. Figure 3 illustrates marketplace makers and actual service providers involved in the marketplace. These provide a set of assertions in XSAL which govern the behavior and execution of standard business processes. Assertions are associated with the standard business processes against which requests are specified. The standard business process are typically provided by the market maker. In exceptional circumstances the market maker could allow the client to provide his/her own business process and then provide additional business rules on top. In Figure 3 a user or client states his/her requests in XSRL. These are combined with the appropriate XSAL assertions and then forwarded to the planning and monitoring framework presented in Figure 4. The planning and monitoring framework interacts with the actual implementations of the services in the service marketplace.

To deal with assertions and user requests we propose a system based on the interleaving of planning and execution. The proposed framework, shown in Figure 4, consists of four components: monitor, planner, executor and run-time support environment and can be seen as an extension of the monitoring framework introduced in [9] to deal with user requests expressed in XSRL.

In this framework the monitor manages the overall process of interleaving planning and execution. It takes user requests, the business process, the business process level as- sertions and starts interacting with the planner. The planner synthesizes a plan and returns it to the monitor. The plan is a sequence of actions to be executed. The planner returns a failure if there was no possible execution satisfying the user request in the given domain without violating the assertions. In case of failure, the monitor eliminates eventual optional goals and assertions or it tries to change service providers. For example, if a business process fails to satisfy the assertion published by one hotel service provider, the framework can try to switch to another hotel service provider whose assertions are less strict. If the planner fails for all possible combinations then the overall execution of the business process fails. If a correct plan exists and therefore it is synthesized. Then the monitor passes it to the executor. The executor is responsible for executing the plan. While executing each action of the plan, the executor may gather new information from the service registry or from the service implementations. Whenever new information is obtained, replanning is potentially needed and the domain updated with the just gathered information is returned back to the monitor. The framework works iteratively until the request is satisfied under the given assertions or there is no successful execution path.

\subsection{Planning domain description}

Using a framework based on interleaved planning and execution demands a formal specification of the business process in terms of planning domains. None of the existing business process definition languages can be used as a domain description for our framework. For example, WS-CDL lacks planning and monitoring mechanisms, BPEL in addition lacks choreography protocol support. The domain representation that we adopt is a state-transition system. It is able to represent non-deterministic actions and potentially 
incomplete knowledge about the environment. Information that is unknown in advance is gathered at run-time by invocations of web services and by contacting the service registry (UDDI) to obtain web service generated information, e.g., current balances, debt histories, etc. Formally, a domain $D$ is a tuple $\langle\mathcal{S}, \mathcal{A}, \mathcal{V}, \mathcal{R}, \mathcal{P}\rangle$, where:

- $\mathcal{S}$ is a set of states a business process can be in.

- $\mathcal{A}$ is a set of actions. An action represents an atomic activity of the business process. Each action is associated with a role. If an action has only one outcome it is called deterministic, it is called nondeterministic otherwise. Action is said to be retractable in a particular state if there exists a sequence of actions that deterministically, independently of the output of the action, will bring back to the state where action was applied preserving the variable values.

- $\mathcal{V}$ is a set of process variables. A variable set includes all the message definitions that are part of the business process. During the execution of the process some of the variables can be undefined. Actions that depend on undefined variables cannot be invoked.

- $\mathcal{R}$ is a set of roles. Roles represent service interfaces that are used in the business process.

- $\mathcal{P}$ is a set of providers. A provider provides a service specification and possibly one or more service implementations. A provider is associated with one of the process roles.

In addition to a domain definition the following relations between the domain items are specified:

- $\rightarrow: \mathcal{S} \times \mathcal{A} \rightarrow 2^{\mathcal{S}}$ is a transition relation between states and actions. A transition represents the application of an action to a state and returns a set of states resulting from that action. Transitions are used to represent the skeleton of the business process control flow. An action can be associated with several transitions.

- role $: \mathcal{A} \rightarrow \mathcal{R}$ is a relation between actions and roles associating a role to each action in the domain. If the service interface is defined in terms of WSDL, the relation is extracted from the port types definition.

- providers $: \mathcal{R} \rightarrow 2^{\mathcal{P}}$ is a relation between roles and providers associating a provider to each role. This mapping is defined in the service registry and typically available to the system only at run-time.

- $f_{a, o}: \mathcal{V} \rightarrow \mathcal{V}$ is a semantic function associated with an action $a$ with an outcome $o$. An outcome can be either 'normal' or 'failure'. For each action, there can be several exception outcomes but there can be only a single normal outcome. All action outcomes are defined with the service interface definition.

\subsection{Service assertion language}

In Section 2.2 we motivated the necessity for having assertions and we classified the operational assertions into simple, preservation, and business entity assertions. The assertions need to be stated in a uniform and unambiguous way by the parties involved in the business process. XSAL (Xml Service Assertion Language) serves this purpose. The syntax of XSAL is defined using BNF notation as follows:

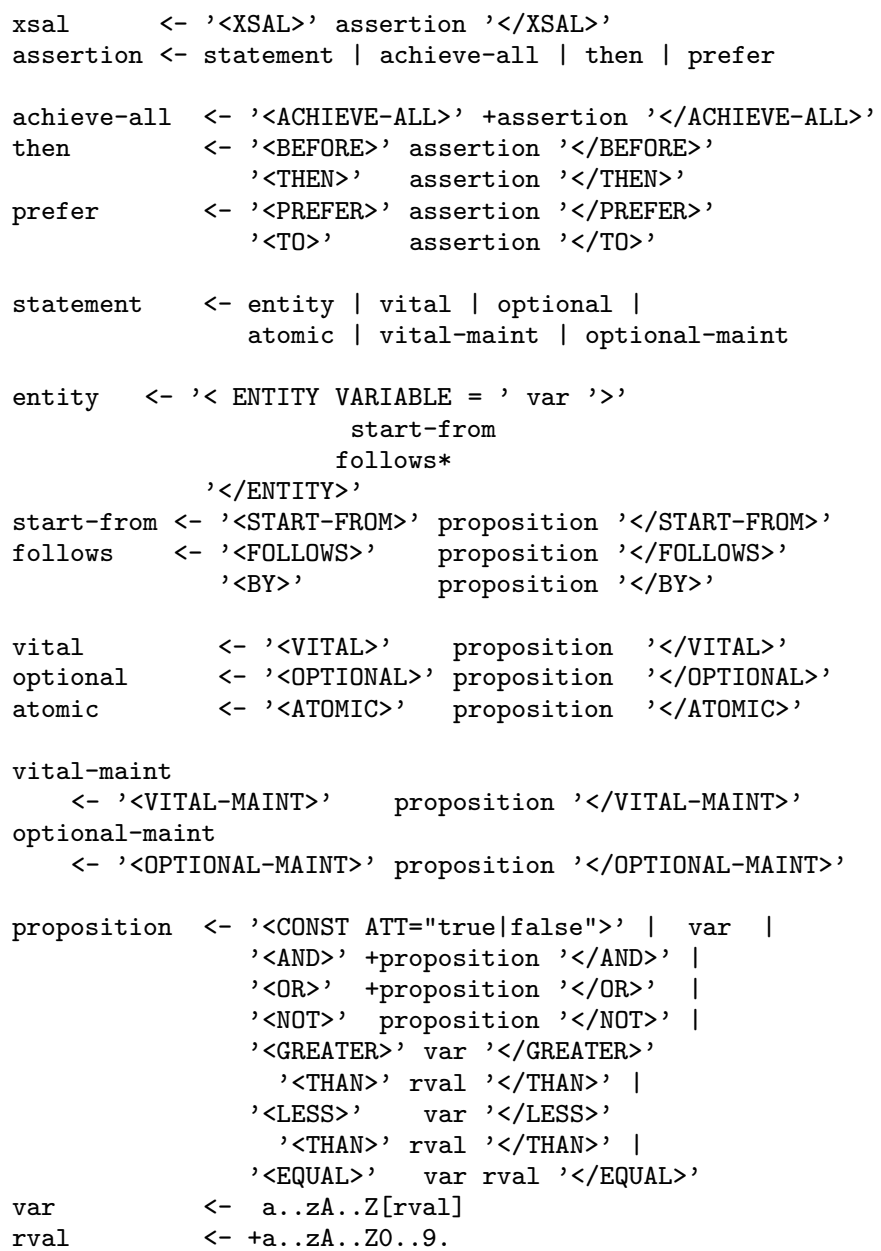

One may observe the similarity between XSAL and XSRL. In fact, these two languages share the same expressive power and interpretation capabilities, though their intended use is quite different. XSAL is used for expressing assertions while XSRL is used for expressing user requests. Before delineating the formal connection among these two languages we shall first provide the intuitive meaning behind XSAL expressions.

The atomic objects of XSAL are propositions, i.e., boolean combination of linear inequalities and boolean propositions. These can be either true or false in any given state. Propositions are further combined by sequencing operators to form assertions. The sequencing operators are: achieve-all, then, prefer. achieve-all succeeds when all nested assertions defined inside the tag <ACHIEVE-ALL> are satisfied, it fails otherwise. The construct then is satisfied when the first statement is satisfied and, from the state where the first statement is satisfied, the second is also satisfied. It fails otherwise. The construct prefer succeeds if the first statement is satisfiable, if not then it succeeds if the second statement is satisfiable, it fails if both statements are unsatisfiable.

The operational assertions can be expressed using the XSAL language. All of the following operators take propositions as arguments. The simple, or reachability, assertions are expressed by XSAL reachability constructs. Formally, reachability constraints require satisfaction of some proposition before execution of the service that has reachability 
assertion. However, strictness of the satisfaction depends on the particular operator. There are three corresponding XSAL operators: atomic, vital, and optional. The atomic operator is used when assertion is strictly important for the party that specifies it and it must be satisfied regardless of nondeterminism or not. More formally, before executing a service that has this type of assertion, constrained propositions must be true. If there is no such execution that makes it true despite of nondeterministic services then the execution fails immediately. The vital operator is used when less strict assertions need to be applied. It tries to find a successful execution to satisfy the constrained proposition. It executes until it has a chance to reach the successful state and fails otherwise. The last operator (optional) is the least strict constraint and demands the satisfaction of the assertion if possible, if not the assertion is just ignored.

XSAL maintainability constructs are employed to express the preservation, or maintainability, assertions. This constructs are used when preservation of some value is needed not only in a single state but during a whole execution sequence. When executing a service with this kind of assertion, only execution paths that preserves the constrained value can be followed. Retractable actions must be handled with care. In fact, if such an action is invoked and later retracted all associated assertions are ignored. As in the case with simple assertions, maintainability assertions can be of different types depending on strictness. We define two types: vital-maint and optional-maint. The first one is used when the proposition value must be preserved along the whole execution path regardless of the nondeterminism. The second (optional-maint) is used when the maintenance assertion is optional.

The entity expression is used to form business entity assertions. This expression begins by relating to a particular variable. It specifies its starting value in the start-from statement and it is continued by any number of follows statements which specify the possible evolutions of the variable. Assertions of this type are always strict.

The semantics of XSAL can be defined following two trajectories: (i) considering formal semantic definition based on execution structures over planning domains as done in [9]; (ii) providing translation rules for transforming XSAL expressions into XSRL and combining them with XSRL expressions. We pursue the second trajectory as it is more intuitive and demonstrates better the relationship between XSAL and XSRL. As a point of notation, we introduce a .t postfix construct to denote the XSAL expression translated into XSRL and (...) to denote the passing of a parameter to a rule, e.g., start-from (var) and follows (var) takes var as a parameter. Expressions where the translation is omitted are propagated unchanged. The symbol ' $*$ ' in the reduction rule denotes the usual Kleene star.

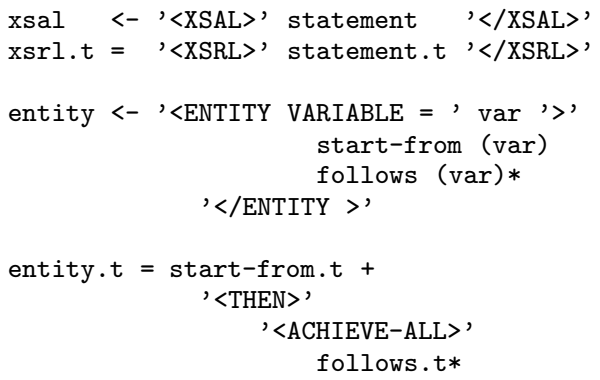

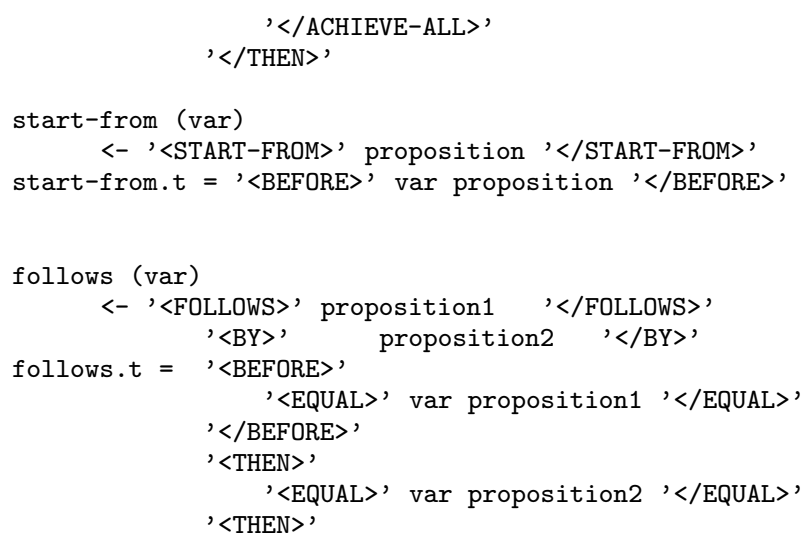

One notes from the translation that the constructs used for propositions, sequencing and expressing preference statements are the same in both languages XSAL and XSRL. The XSAL business entity assertion construct is not present in XSRL and is translated into the sequencing operators before-then binding the business entity variable to propositions.

\subsection{A domain instance}

In the following we revisit the traveling domain example shown in Figure 1 to explain the use of XSAL and associated constructs. Next we present it according to the formal definition of a domain $D$ presented in Section 3.1.

There are fourteen states $\mathcal{S}=\{1,2, \ldots, 14\}$ in the upper half of the figure. The set of variables $V$ ar is $\{$ hotelReserved, hotelPrice, location, trainBooked, trainPrice, flightBooked, flightPrice, confirmed, money\}, among which one distinguishes the boolean variables (hotelReserved, trainBooked, flightBooked, confirmed), from the real variables (hotelPrice , trainPrice, flightPrice, money), and a variable representing location names (location). In the set of variables a subset is defined to be of knowledge variables. In the example, we define hotelPrice, trainPrice, flightPrice to be knowledge variables. There are also nineteen actions that can be performed in the domain $A c t=\left\{a_{1}, \ldots, a_{19}\right\}$.

Several roles are involved in the travelling business process, that is, $\mathcal{R}=$ user, hotel, payment, insurance, train, air $\}$. The user role represents the requesting party. Typically, it is a human user but it could also be any application software utilizing the business process. The set of actual providers for the roles $\mathcal{R}$ are stored in the service registry.

Arrows in Figure 1 represent process actions. For example, states (3) and (4) are connected by the reserveHotel action of the hotel role. This has two outcomes: normal, where the variable hotelReserved is set to true and exception, where the hotel remains unreserved. This action is an example of a nondeterministic action. The two arrows from the state (4) represent different outcomes for this action. Other examples of actions are bookflight for the air role and getTrainPrice for train role.

Assertions work in conjunction with the travelling business process and are defined in XSAL. The business process level assertion that ensures that the process always reaches the final state is expressed in the following way: atomic final. In the following we omit XML tags brevity. Examples of a role-level assertions are requirement for insurance in case of long vacations: vital (healthRisk $\rightarrow$ insuranceTaken), where $\rightarrow$ represents logical implication and is expressed us- 
ing the $<\mathrm{NOT}>$ and $<\mathrm{OR}>\mathrm{XSAL}$ expressions, as usual.

At the provider level, the hotel provider may prefer, for example, a specific credit card type for payment: optional cardType $=$ VISA.

The maintenance assertion for customers of royalty services that was introduced in Section 3.1 is encoded as follows: optional royaltyCard $\rightarrow$ (roleType $=$ acceptsRoyal tyCard)).

In the following we use XSAL to codify the business entity assertion that was presented in Figure 2. The XSAL syntax for this assertion is:

entity travelPackage

start-from requested

follows requested by rejected $\vee$ accepted by travel agency

follows rejected by requested

follows accepted by travel agency by rejected $\vee$ approved by client

follows approved by client by package completed

Additional details like precise hotel information, seats type, payment numbers, etc. can be easily integrated in the above example. To do so, one should add corresponding variables and modify the semantic functions of the actions to take into account the variables introduced. We omit such additional details herein to improve readability.

\subsection{Planning and monitoring algorithms}

in the previous we have introduced a planning and monitoring framework and the assertion language XSAL. In this section, we present the algorithms which handle XSAL assertions together with XSRL requests. The algorithms presented are an extension of the ones based on interleaving planning and execution introduced to handle XSRL requests in [9]. Referring to Figure 4, we recall that the framework consists of three main components, that is, a monitoring, a executor and a planner. We present algorithms for these components in the following.

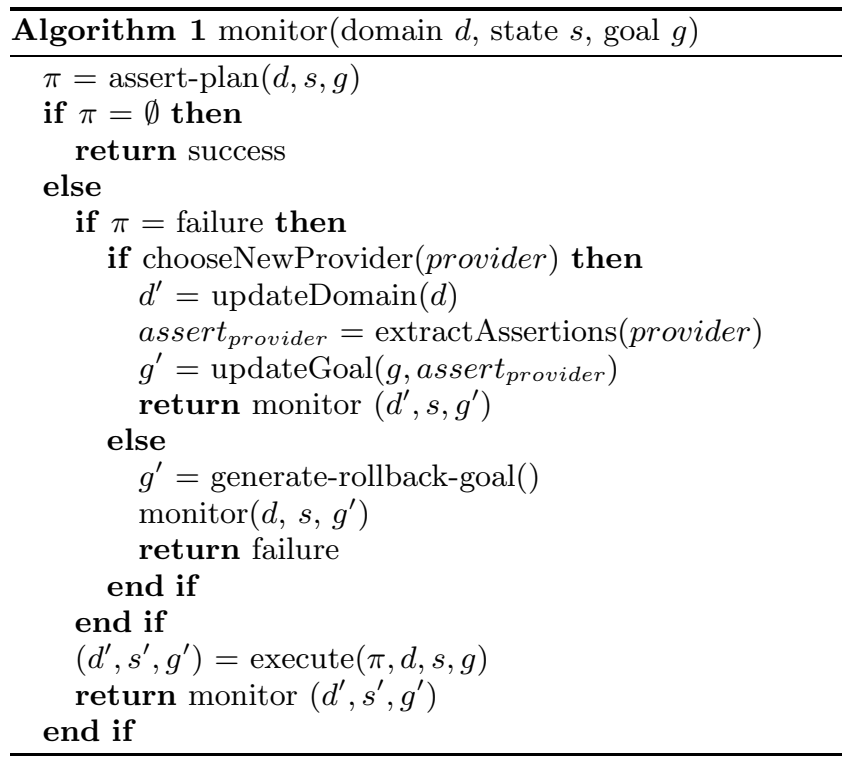

The monitor takes a domain $d$, that is built on the basis of the business process, an initial state $s$ and a goal $g$. The initial request of the user to the system is combined together with business process assertions, thus, the monitoring algorithm is invoked initially with the following goal: achieve-all(request, assert $_{b p}$ ), where request is the user request and assert $_{b p}$ is the set of business process level assertions.

The monitor (Algorithm 1) is the core of the interleaved planning and execution process. It invokes the planner and the executor in order to satisfy the user requests and the assertions, and it recovers from failures. The algorithm is an extension of the monitoring algorithm presented in [9], where the most notable difference is the updating of the goal to take into account the provider level assertions. When a new provider is chosen then the goal is modified in the following way. First, assertions that are associated with the previously assigned provider being deassigned are eliminated from the goal. Second, assertions of the new provider are added to a goal by using the achieve-all operator. The modification of the goal to take assertions into account is performed by the extractAssertions and updateGoal functions.

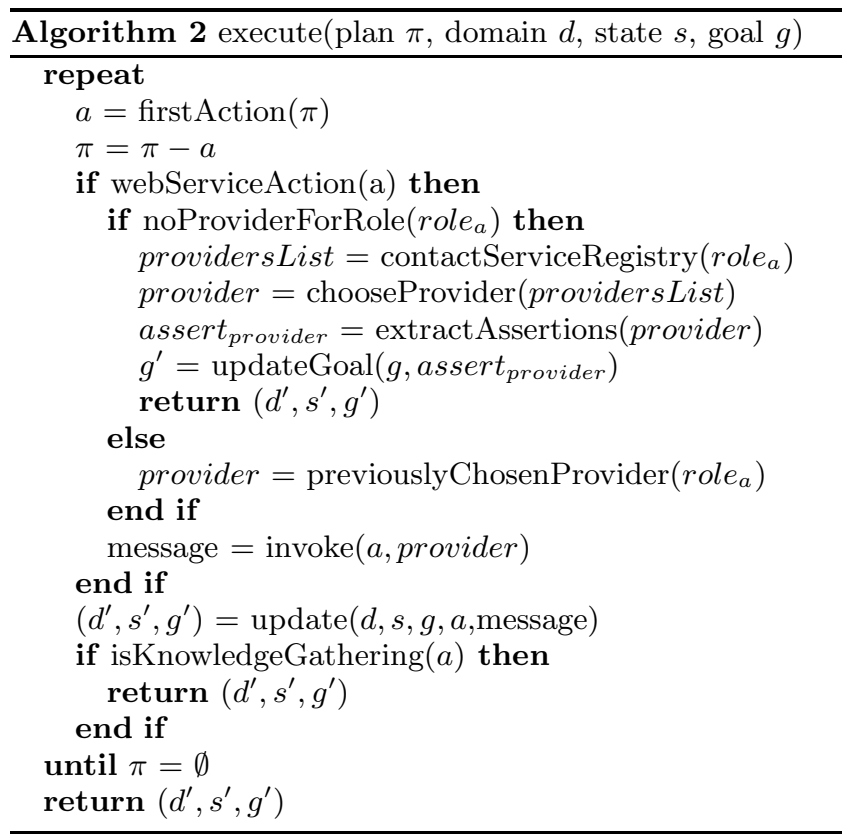

The executor (Algorithm 2) takes a plan and executes it in the marketplace. It contacts the service registry when a service implementation for a given role is necessary, it executes actions of the plan and it checks whether replanning is required. When a new provider is requested from the service registry, its assertions are added to the goal $g$ in the following way achieve-all $\left(g\right.$, assert $\left.t_{\text {provider }}\right)$. This is achieved via the extractAssertions and updateGoal functions.

The planning algorithm is presented in two parts: one dealing with role level assertion and one actually synthesizing a plan. The assert-planner (Algorithm 3) checks validity of role-level assertions. The assert-planner works in the following way. First, it produces an initial plan by invoking the plan function (Algorithm 4). If the planner succeeds by producing a plan then the assert-planner checks if the plan contains actions with new assertions. If it does, then all assertions are added to the goal and replanning is requested. If the planner fails to synthesize a plan then the assert-planner marks all actions that possibly violate the plan as optional goals and request replanning. Optional goals are added to the current goal $g$ in the following way: 


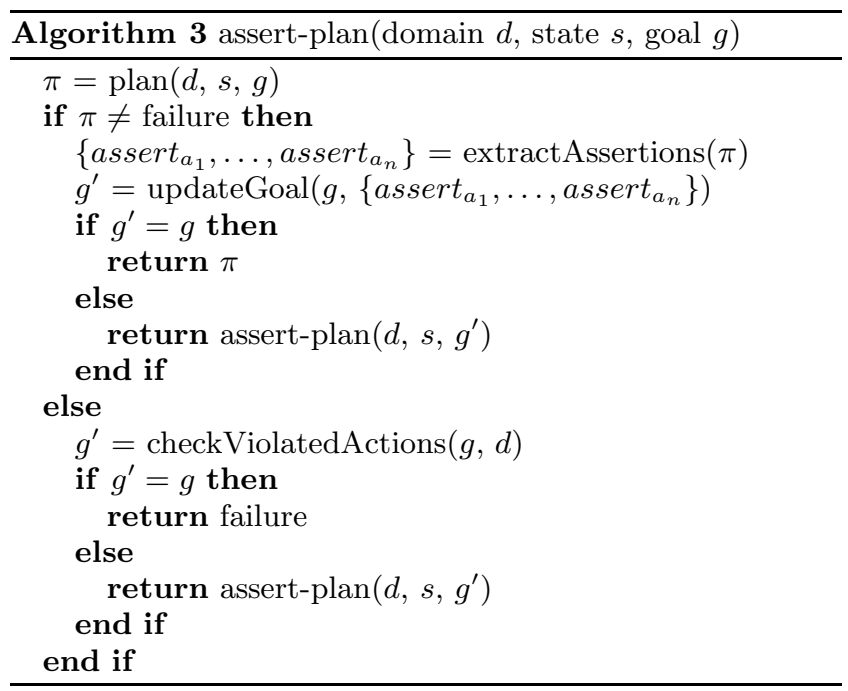

$g^{\prime}=\operatorname{achieve-all}\left(g\right.$, optional $\neg a_{1}, \ldots$, optional $\left.\neg a_{n}\right)$, where optional $\neg a_{i}$ indicates that the action $a_{i}$ should be avoided, if possible. The assert-planner returns a plan if the user request and all assertions are satisfied and failure otherwise.

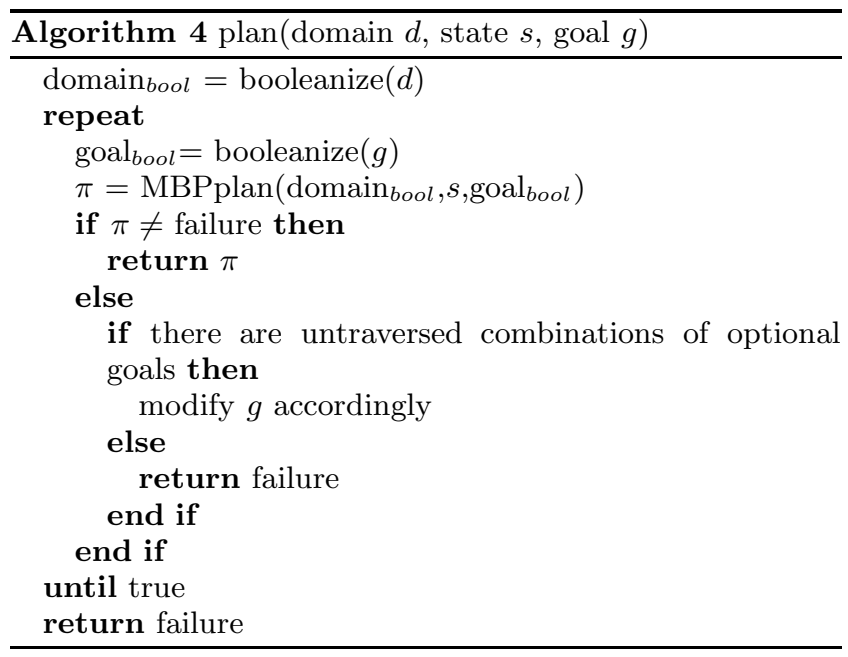

The planner (Algorithm 4) is based on the existing planner based on model checking [3] and is proposed as in [9]. The planner is responsible for synthesizing a plan based on a given domain $d$, an initial state $s$ and a goal $g$. The planner returns a plan if it exists and failure otherwise. The planner checks all possible combinations of optional goals before returning a failure.

\section{A MONITORING EXAMPLE}

To illustrate the application of the algorithms presented in the context of the planning and monitoring framework, we use the example presented in section 2.1 and formalized in section 3.3. Suppose that a user is planning a trip to Nowhereland and is interested in a number of possibilities in connection with this trip. These include making a hotel reservation, avoiding to travel by train, if possible, and spending an overall amount not greater than 300 euro for the whole package. Further, the user may prefer to spend less than 100 euro for a hotel room, if possible. However, if this is not possible, the user is not willing to spend more than
200 euro for that room. This is expressed by the following XSRL request:

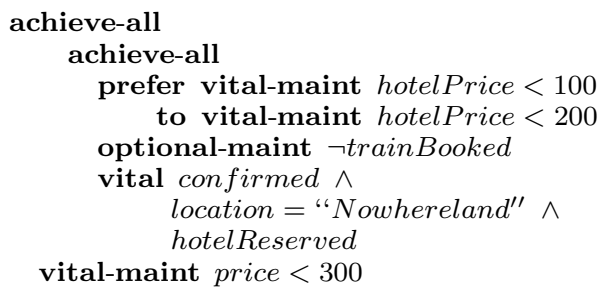

In addition, assume that two independent XSAL business process level assertions such as the business entity assertion in Figure 2 (state diagram) and a atomic final are published. The first assertion specifying the various states in which a travel package can go through, and the second forcing the transactions to always completing atomically. In the given example, the second assertion forces the package to go to Nowhereland, once booked, to be bought or rejected entirely.

The system starts by combining the user request with the business process assertions in an achieve-all construct. The monitor invokes the assert-planner which in turn invokes the planner. With the above goal, business process assertion and domain as shown in Figure 1, the initial plan provided by the planner is the following sequence of actions: getHotelPrice, reserveHotel, and so on.

The monitor then sends the plan to the executor to start interacting with web service implementations. By these invocation a travel agency and a hotel provider are selected and a room is reserved. Suppose that the government considers Nowhereland to be a health risky location. Then the role level assertion vital (healthRisk $\rightarrow$ insuranceTaken) coming from the service registry together with the travel agency role is considered. At this point, the executor returns control to the monitor which in turn requests a new plan from the assert-planner taking into account the given rolelevel assertion. The new plan generated will now comprise an action bringing the process in the obtained a medical insurance state.

Suppose further that the selected hotel is "MyHotel" which comes with the provider level assertion optional cardType $=$ VISA. Then, when the executor runs the request payment from the user the cardType is asked to be VISA. If the user refuses such option, execution can nevertheless proceed given the optionality of the assertion. Note that if the assertion was vital cardType = VISA then the user's refusal would result in a assertion violation and thus a plan failure.

As for a maintainability assertion, suppose that the travel agency is asked by the client to to provide services complying with a given royalty card. Therefore, the travel agency publishes the following assertion: optional(royaltyCard $\rightarrow$ $($ roleType $=$ acceptsRoyaltyCard $))$. This is taken into account by the assert-planner as soon as the user has specified the card in his request.

When a business entity assertion requires a travel package is to be assembled following specific business rules such as the ones in Figure 2, this assertion is always taken into account by the assert-planner when providing new plans to the monitor. Finally, the execution proceeds until the travel package is completed and the user approval is requested. At this point the business level assertion atomic final is the last to be satisfied. This is achieved by a plan going to the final state of the business process. 


\section{RELATED WORK}

In the web services literature there are several approaches dealing with the monitoring of the assertions over serviceenabled business processes. The WS-Policy framework [18] provides a general purpose model for describing a broad range of service requirements, preferences, and capabilities. Typically, it is used when the provider describes the set of conditions the requester should satisfy before invoking the service. RuleML [5] is a powerful technique for expressing business rules over semantically annotated service. On the negative side is the lack of any support for run-time monitoring of the business rules.

With respect to web services and planning techniques a review of web service composition techniques is presented in [17] and it is argued that planning techniques can be of help in tackling the problem of web service composition. Temporally extended goals, i.e., goals expressing not only desired states to achieve but also conditions on how these are to be reached, are on expressive way of defining complex business goals $[13,9,15]$. Various authors have emphasized the importance of planning for web services $[8,10,11]$. In particular, Knoblock et al. [8] use a form of template planning based on hierarchical task networks and constraint satisfaction, in [10] regression planning is used, while in [11] the Golog planner is used to automatically compose semantically described services. Various authors use planners over service description in DAML-S [16, 17]. Feasibility of HTN planning algorithms was shown in [19]. Finite-state machine framework for automatic composition was introduced in [2]. Service orchestration based on object-oriented data models are presented in [4]. In [12] service composition rules are used for governing the business process construction.

\section{CONCLUDING REMARKS}

In our previous work we developed a service request language and support framework that allow users to formulate their requests against standard business processes $[1$, $13,9]$. In this paper we extend this approach by presenting a framework capable of automatically associating business rules with relevant processes involved in a user request. More specifically, we have introduced the assertion language $\mathrm{XSAL}$ whose main purpose is to express business rules in the form of assertions over business processes. This allows for consistency and conformance to organizational rules and policies when executing a business process. Additionally, it offers run-time control over its execution. We have classified assertions with respect to two process characteristics: its operational context and its ownership. As regards the operational context, we distinguish between simple, preservation and business entity assertions. As regards ownership, we distinguish between business process, role and provider level assertions. We have then introduced a framework for planning user requests that comply with assertions and monitoring their execution to recover from violating conditions. Specialized algorithms for planning, monitoring and executing requests and assertions have been proposed for this framework.

The proposed framework and the XSAL language open several interesting research issues. A particularly interesting open issue regards the performance of the framework, in particular, the way providers are selected from the service registry is crucial for the efficiency and effectiveness of the architecture. The current proposal does not address this issue, in other words, providers are chosen randomly. A better solution would be that of selecting providers based on provider-level assertions (for instance by comparing active assertions), on reputation and history of previous interactions with the provider, or optimizing some specific QoS parameter (e.g., cost of the service, average latency of the service, etc.).

The proposed framework plans for requests and assertions, then monitoring the execution of the plans. If there is one possible execution path that can satisfy the request and comply with its associated assertions, this will be found and executed, if not, a failure will be returned. In case that a request succeeds no information is currently provided regarding the quality of the execution. That is, if more possible execution paths complying with the assertions and the user request exist, then only one is guaranteed to be taken. An open issue concerns the comparison of potential solutions (execution trajectories) against optimality metrics, e.g., the shortest plan, the cheapest, the fastest or any other optimality criteria.

\section{REFERENCES}

[1] M. Aiello, M. Papazoglou, J. Yang, M. Carman, M. Pistore, L. Serafini, and P. Traverso. A request language for web-services based on planning and constraint satisfaction. In $V L D B$ Workshop on Technologies for E-Services (TESO2), 2002.

[2] D. Berardi, D. Calvanese, G. D. Giacomo, and M. Mecella. Reasoning about Actions for e-Service Composition. In Proceedings of ICAPS'03 Workshop on Planning for Web Services, Trento, Italy, June 2003.

[3] P. Bertoli, A. Cimatti, M. Pistore, M. Roveri, and P. Traverso. MBP: A Model Based Planner. In Proc. IJCAI'01 Workshop on Planning under Uncertainty and Incomplete Information, 2001.

[4] I. Fikouras and E. Freiter. Service discovery and orchestration for distributed service repositories. In Conf. on Service-Oriented Computing (ICSOC-03), Lecture Notes in Computer Sciences, pages 59-74. Springer, 2003.

[5] B. N. Grosof. Representing e-commerce rules via situated courteous logic programs in ruleml*1. Electronic Commerce: Research and Applications, 3(1):2-20, 2004.

[6] P. Harmon. Analyzing activities. Business Process Trends, 1(4), 2003.

[7] Kavantzas. Web Services Choreography Description Language 1.0, April 2004. http://lists.w3.org/ Archives/Public/www-archive/2004Apr/att-0004/ cdl_v1\%-editors-apr03-2004-pdf .pdf.

[8] C. A. Knoblock, S. Minton, J. L. Ambite, M. Muslea, J. Oh, , and M. Frank. Mixed-initiative, multi-source information assistants. In Proceedings of the World Wide Web Conference, 2001.

[9] A. Lazovik, M. Aiello, and M. Papazoglou. Planning and monitoring the execution of web service requests. In Conf. on Service-Oriented Computing (ICSOC-03), Lecture Notes in Computer Sciences 2910, pages 335-350. Springer, 2003.

[10] D. McDermott. Estimated-regression planning for 
interactions with Web Services. In $6^{\text {th }}$ Int. Conf. on AI Planning and Scheduling. AAAI Press, 2002.

[11] S. McIlraith and T. C. Son. Adapting Golog for composition of semantic web-services. In D. Fensel, F. Giunchiglia, D. McGuinness, and M. Williams, editors, Conf. on principles of Knowledge Representation (KR), 2002.

[12] B. Orriens, J. Yang, and M. Papazoglou. Model driven service composition. In Conf. on Service-Oriented Computing (ICSOC-03), Lecture Notes in Computer Sciences, pages 75-90. Springer, 2003.

[13] M. Papazoglou, M. Aiello, M. Pistore, and J. Yang. Planning for requests against web services. IEEE Data Engineering Bulletin, 25(4):41-46, 2002.

[14] M. P. Papazoglou and D. Georgakopoulos. Service-oriented computing. Commun. ACM, 46(10):24-28, 2003.

[15] M. Pistore, F. Barbon, P. Bertoli, D. Shaparau, and P. Traverso. Planning and Monitoring Web Service Composition . In ICAPS'04 Workshop on Planning and Scheduling for Web and Grid Services, June 2004.
[16] M. Sheshagiri, M. desJardins, and T. Finin. A Planner for Composing Services Described in DAML-S. In Proceedings of ICAPS'03 Workshop on Planning for Web Services, Trento, Italy, June 2003.

[17] B. Srivastava and J. Koehler. Web Service Composition - Current Solutions and Open Problems. In Proceedings of ICAPS'03 Workshop on Planning for Web Services, Trento, Italy, June 2003.

[18] WS-Policy. Web Services Policy Framework, May 2003. http://www-106.ibm.com/developerworks/ library/ws-polfram/.

[19] D. Wu, E. Sirin, J. Hendler, D. Nau, and B. Parsia. Automatic Web Services Composition Using SHOP2. In Proceedings of ICAPS'03 Workshop on Planning for Web Services, Trento, Italy, June 2003. 